\title{
可驯表示型与野表示型 bocs*
}

\author{
徐运阁** \\ 张英伯 \\ (湖北大学数学与计算机科学学院, 武汉 430062) \\ (北京师范大学数学科学学院, 北京 100875)
}

摘要简化了 Drozd 关于极小野 bocs 的一个著名定理的证明, 这使得该证明 中所构造的矩阵的阶数由 43 降低到 20. 为了刻画可刊表示型 bocs 的微分, 给出 了所有可能的极小野 bocs, 并讨论了可刊表示型 bocs 的首箭的微分形式.

\section{关键词 bocs 可驯表示型 野表示型 微分}

\section{1 引言}

设 $k$ 是代数闭域， $\Lambda$ 是有限维结合 $k$-代数，且含有单位元 1. 著名的 Drozd Tame-Wild 定理 ${ }^{1]}$ 断言 $\Lambda$ 或者是可驯表示型, 或者是野表示型, 二者只居其一 且必居其一. 这证明了 P. Donovan 与 M.R. Freislich 的一个著名猜想. 该定理的 证明依赖于 bocs 的概念. bocs 是由 A.V. Roiter ${ }^{[2]}$ 为阐述表示论中的矩阵方法 与微分分次范畴的方法而引入的一个代数结构. 基于这一概念及其约化技巧, Crawley-Boevey 证明了对任意可驯表示型代数 $\Lambda$, 给定正整数 $d$, 几乎所有的维数 至多为 $d$ 的不可分解 $\Lambda$-模都与它的 AR- 平移同构, 因此都在齐次管上，从而对 可驯表示型代数的 AR- 箭图给出了一个较精细的刻画.

$\mathcal{A}=(A, V)$ 称为 bocs, 如果 $A$ 是骨架小范畴, 且 $V$ 是 $A$ - 余代数. 这样 $V$ 是一个具有余代数结构的 $A-A$ - 双模, 其中余单位及余乘分别由 $\varepsilon: V \rightarrow A$ 和 $\mu: V \rightarrow V \otimes_{A} V$ 给出. $\varepsilon$ 的核记为 $\bar{V}$. $\mathcal{A}$ 的表示范畴 $R(\mathcal{A})$ 与从 $A$ 到 $\bmod (k)$ 的反 变函子范畴 $R(A)$ 具有相同的对象; 设 $M, N$ 是 $\mathcal{A}$ 的两个表示, 则从 $M$ 到 $N$ 的态 射由从 $V \otimes_{A} M$ 到 $N$ 的 $A$ - 模同态给出. 态射 $\theta: V \otimes_{A} M \rightarrow N$ 与 $\phi: V \otimes_{A} N \rightarrow L$ 的合成 $\theta \phi$ 定义为

$$
V \otimes_{A} M \stackrel{\mu \otimes 1}{\longrightarrow} V \otimes_{A} V \otimes_{A} M \stackrel{1 \otimes \theta}{\longrightarrow} V \otimes_{A} N \stackrel{\phi}{\longrightarrow} L .
$$

2004-09-21 收稿

* 国家自然科学基金 (批准号: 19701030, 10201004) 和湖北省教育厅重大项目基金资助项目

** E-mail: xuy@hubu.edu.cn 
本文仅考虑分层 (layered) bocs, 参见文献 [3] 定义 3.6.

矩阵问题有多个等价的表述形式 ${ }^{[4]}$, 如 bocs, 微分分次范畴 ${ }^{[2]}$, 微分双箭图, 双模问题 ${ }^{[5]}$, 相对投射范畴 ${ }^{[6]}$ 等. 其中 bocs 的最大优势在于它的约化技巧, 例如 它在 Drozd 定理的证明中提供了归纳步骤. 定理 3.2(参见文献 [1] 命题 9 或文献 [3] 命题 3.10) 在 Drozd 定理的证明中是至关重要的, 它保证了某些 “坏” 的分支 不会出现在可驯 bocs 中. 众所周知, 如果 $\mathcal{A}$ 是野表示型的, 那么必定在某一步约 化中将会遇到定理 3.2 中所列出的极小野 bocs 之一. 本文首先简化了 Drozd 关于 极小野 bocs 的一个著名定理的证明, 这使得该证明中所使用的矩阵的阶数由 43 降低到 20. 我们也注意到定理 3.2 中所列出的极小野 bocs 不是完备的, 因此为了 刻画可驯表示型 bocs, 我们也给出了尽可能多的极小野 bocs, 并讨论了可驯表示 型 bocs 的首箭的微分形式. 这些结果对可驯表示型代数的研究是很有帮助的, 也将有助于有限维代数的野秩 (wild-rank) 的计算, 而后者与著名的 Tame-open 猜 想密切相关 ${ }^{1)}$.

本文总假定 $k$ 是代数闭域，箭向或态射的合成总是从左到右.

\section{2 首箭的微分}

固定一个 bocs $\mathcal{A}=(A, V)$ 和它的一个层 $L=\left(A^{\prime} ; \omega ; a_{1}, \cdots, a_{n} ; v_{1}, \cdots, v_{m}\right)$. 由于 bocs 的最大优势在于它的约化技巧, 这为它在许多的具体应用中 (如 Drozd 定理的证明) 提供了归纳步骤. 因而有必要首先讨论第 1 个箭向 $a_{1}$ 的微分. $a_{1}$ 将 会有如下 4 种可能:

(1) $\mathcal{P}=\mathcal{Q}, \quad \lambda \bigcirc \stackrel{\mathcal{P}}{\bullet} a_{1} \cdot A^{\prime}(\mathcal{P}, \mathcal{P})=k\left[\lambda, f(\lambda)^{-1}\right]$, 其中 $f(\lambda) \in k[\lambda]$.

(2) $\mathcal{P} \neq \mathcal{Q}, \quad \lambda \bigcirc \stackrel{\mathcal{P} a_{1}}{\mathcal{Q}} \bigcirc \mu . \quad A^{\prime}(\mathcal{P}, \mathcal{P})=k\left[\lambda, f(\lambda)^{-1}\right], A^{\prime}(\mathcal{Q}, \mathcal{Q})=$ $k\left[\mu, g(\mu)^{-1}\right]$, 其中 $f(\lambda) \in k[\lambda], \quad g(\mu) \in k[\mu]$.

(3) $\mathcal{P} \neq \mathcal{Q}, \quad \lambda \bigcirc \stackrel{\mathcal{P} \quad a_{1} \longrightarrow \mathcal{Q}}{\longrightarrow} \mathcal{Q}\left(\right.$ 相应地, $\left.\stackrel{\mathcal{P}}{\longrightarrow} a_{1} \stackrel{\mathcal{Q}}{\longrightarrow} \bigcirc \mu\right)$ ). $A^{\prime}(\mathcal{P}, \mathcal{P})=k\left[\lambda, f(\lambda)^{-1}\right]\left(\right.$ 相应地, $A^{\prime}(\mathcal{Q}, \mathcal{Q})=k\left[\mu, g(\mu)^{-1}\right]$, 其中 $f(\lambda) \in k[\lambda]($ 相应 地, $g(\mu) \in k[\mu])$.

(4) $a_{1} \bigcap_{\boldsymbol{r}} \cdot \mathcal{P}$ 或 $\underset{\mathcal{P}}{\stackrel{a_{1}}{\longrightarrow}} \stackrel{a^{\mathcal{Q}}}{\longrightarrow} \cdot A^{\prime}(\mathcal{P}, \mathcal{P})=A^{\prime}(\mathcal{Q}, \mathcal{Q})=k$.

对于情形 (1) 和 (2),

$$
\delta\left(a_{1}\right)=\sum_{j=1}^{m} h_{j}(\lambda, \mu) v_{j},
$$

1) Han Yang. Is tame open? arXiv:math. RT/0403087v1 
其中 $\lambda v_{j}$ 表示用 $\lambda$ 左乘以 $v_{j}$, 而 $\mu v_{j}$ 在第 1 种情形表示用 $\lambda$ 右乘以 $v_{j}$, 在第 2 种情 形表示用 $\mu$ 右乘以 $v_{j}$.

设 $h(\lambda, \mu)$ 是 $h_{j}(\lambda, \mu)(j=1, \cdots, m)$ 的最大公因式, $q_{j}(\lambda, \mu)=h_{j}(\lambda, \mu) / h(\lambda, \mu)$. 由于 $q_{j}(\lambda, \mu)$ 互素, 故存在多项式 $s_{j}(\lambda, \mu)$ 及非零多项式 $c(\lambda)$, 使得在多项式环 $k[\lambda, \mu]$ 中,

$$
c(\lambda)=\sum_{j=1}^{m} s_{j}(\lambda, \mu) q_{j}(\lambda, \mu),
$$

因而在 Hermite 环 $S=k\left[\lambda, \mu, f(\lambda)^{-1}, g(\mu)^{-1}, c(\lambda)^{-1}\right]$ 中，有

$$
1=\sum_{j=1}^{m} c(\lambda)^{-1} s_{j}(\lambda, \mu) q_{j}(\lambda, \mu)
$$

故存在可逆矩阵 $Q \in M_{m \times m}(S)$, 它的第 1 行元素为 $\left(q_{j}(\lambda, \mu)\right)_{j=1, \cdots, m}$. 作基变换

$$
\left(w_{1}, w_{2}, \cdots, w_{m}\right)^{\mathrm{T}}=Q\left(v_{1}, v_{2}, \cdots, v_{m}\right)^{\mathrm{T}},
$$

使得

$$
\delta\left(a_{1}\right)=h(\lambda, \mu) w_{1}
$$

对于情形 (3),

$$
\delta\left(a_{1}\right)=\sum_{j=1}^{m} h_{j}(\lambda) v_{j}
$$

设 $h(\lambda)$ 是 $h_{j}(\lambda)(j=1, \cdots, m)$ 的最大公因式, 则 $q_{j}(\lambda)=h_{j}(\lambda) / h(\lambda)$ 互素, 故存 在多项式 $s_{j}(\lambda) \in k[\lambda]$, 使得 $1=\sum_{j=1}^{m} s_{j}(\lambda) q_{j}(\lambda)$. 类似地经过基变换可得

$$
\delta\left(a_{1}\right)=h(\lambda) w_{1} .
$$

对于情形 (4), 经过 $S=k$ 上的基变换可得

$$
\delta\left(a_{1}\right)=w_{1}, \text { 或 } \delta\left(a_{1}\right)=0 .
$$

\section{3 极小野 bocs}

本节给出 Drozd 关于极小野 bocs 定理的一个简化证明，这使得该证明中所 使用的矩阵的阶数由 43 降低到 20. 该证明依赖于 Belitskii 算法. 这是一个将单 个矩阵约化为相似变换下的典范形的有效算法, 该典范形可看做 Jordan 标准形 的推广 (参见文献 [7]).

定义 3.1 ${ }^{[3]}$ Bocs $\mathcal{A}=(A, V)$ 称为野表示型的，如果存在既保持同构类又保 持不可分解性的函子 $F: k\langle x, y\rangle-\bmod \rightarrow R(\mathcal{A})$, 否则 $\mathcal{A}$ 称为是可驯表示型的.

我们重新表述 Drozd 关于极小野 bocs 的定理如下:

www.scichina.com 
定理 3.2 ${ }^{[1,3]}$ 设 $\mathcal{A}=(A, V)$ 是一个 bocs, $A^{\prime}$ 仅有不可分解对象 $\mathcal{P}$ 与 $\mathcal{Q}$, 则 $\mathcal{A}$ 在下述情形下必是野表示型的:

情形 1. $\delta\left(a_{1}\right)=h(\lambda, \mu) w_{1}, h(\lambda, \mu) \in k\left[\lambda, \mu, f(\lambda)^{-1}, g(\mu)^{-1}\right]$ 是不可逆的，
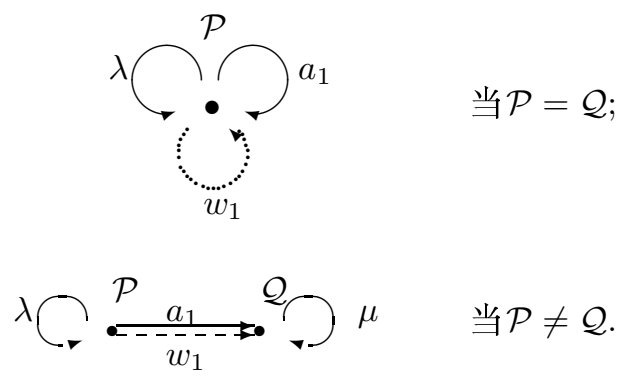

情形 2. $\delta\left(a_{1}\right)=0, A^{\prime}(\mathcal{P}, \mathcal{P})=k\left[\lambda, f(\lambda)^{-1}\right]\left(\right.$ 相应地 $\left.A^{\prime}(\mathcal{Q}, \mathcal{Q})=k\left[\mu, g(\mu)^{-1}\right]\right)$,

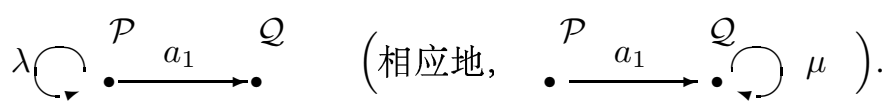

注 3.3 为了证明 $\mathcal{A}$ 是野表示型的，必须构造一个既保持同构类又保持不 可分解性的函子

$$
F: k\langle x, y\rangle-\bmod \rightarrow R(\mathcal{A}) .
$$

该函子的构造依赖于由 bocs 的微分所引起的各种关系. 为了避免对这些关系过 分繁琐而又冗长的技巧化的分析，我们采用自由参数化典范矩阵来构造这个函 子. 粗略地说, 设 $M \in R(\mathcal{A})$ 是一个不可分解的自由参数化典范矩阵, 它包含两 个代数无关的自由参数 $\xi, \eta$, 则 $M$ 诱导了函子

$$
F_{M}: k\langle x, y\rangle-\bmod \rightarrow R(\mathcal{A}),
$$

它将维数为 $m$ 的表示 $L=(L(x), L(y)) \in k\langle x, y\rangle-\bmod$ 映为矩阵 $M^{m}(L(x), L(y))$, 该矩阵是将矩阵 $M$ 的常值元素 $a$ 扩大为 $a I_{m}$, 将自由参数 $\eta$, $\xi$ 分别改变为 $L(x)$, $L(y)$ 而得到的, 这仍然是 $R(\mathcal{A})$ 中的不可分解典范矩阵 ${ }^{[8]}$. 而且, 表示 $M^{m}(L(x)$, $L(y))$ 的自同态环由上三角分块矩阵组成, 其对角块是 $\operatorname{End}_{k\langle x, y\rangle}(L)$, 因此诱导函 子 $F_{M}$ 保持不可分解性与同构类 (详细内容参见 Zhang 等 ${ }^{1)}$ 文章中的文献).

定理 3.2 的证 设 $M$ 是 $\mathcal{A}$ 的维数向量为 $\underline{m}=\left(m_{\mathcal{P}}, m_{\mathcal{Q}}\right)$ 的一个不可分解表 示, $M(\mathcal{P})=k^{m_{\mathcal{P}}}, M(\mathcal{Q})=k^{m_{\mathcal{Q}}}$. 选择这些向量空间的一组合适的 $k$ - 基, 我们 可以将 $M(\lambda)=W^{\lambda}, M(\mu)=W^{\mu}$ 取作 Weyr 矩阵 ${ }^{[7]} . M$ 的自同构由可逆矩阵 $S_{\mathcal{P}}$ 和 $S_{\mathcal{Q}}$, 以及矩阵 $S\left(w_{1}\right)=\left(v_{i j}\right)_{m_{\mathcal{P}} \times m_{\mathcal{Q}}}$ 给出, 它们满足

$$
\begin{gathered}
S_{\mathcal{P}} M(\lambda)-M(\lambda) S_{\mathcal{P}}=0, \quad S_{\mathcal{Q}} M(\mu)-M(\mu) S_{\mathcal{Q}}=0 \\
M\left(a_{1}\right) S_{\mathcal{Q}}-S_{\mathcal{P}} M\left(a_{1}\right)=S\left(\delta\left(a_{1}\right)\right)=M(h(\lambda, \mu)) S\left(w_{1}\right) .
\end{gathered}
$$

1) Zhang Yingbo, Xu Yunge. Tameness and homogeneity 
根据这些关系, $M\left(a_{1}\right)$ 由 Belitskii 约化算法可以取作典范形. 设

$h(\lambda, \mu)=\alpha\left(\lambda-\lambda_{0}\right)+\beta\left(\mu-\mu_{0}\right)+\gamma_{1}\left(\lambda-\lambda_{0}\right)^{2}+\gamma_{2}\left(\lambda-\lambda_{0}\right)\left(\mu-\mu_{0}\right)+\gamma_{3}\left(\mu-\mu_{0}\right)^{2}+\cdots$

以下 $D$ 表示参数 $\{\lambda, \mu\}$ 的定义域. 下面将根据不同的情形分别构造含有两个代 数无关的自有参数的不可分解矩阵 $M$.

情形 $1(2), \beta \neq 0$.

令 $m_{\mathcal{P}}=9, m_{\mathcal{Q}}=11,\left(\lambda_{0}, \mu_{0}\right) \in D$ 满足 $h\left(\lambda_{0}, \mu_{0}\right)=0, f\left(\lambda_{0}\right) \neq 0, g\left(\mu_{0}\right) \neq 0$. $M(\lambda) \simeq J_{1}\left(\lambda_{0}\right) \oplus J_{3}\left(\lambda_{0}\right) \oplus J_{5}\left(\lambda_{0}\right), M(\mu) \simeq J_{2}\left(\mu_{0}\right) \oplus J_{4}\left(\mu_{0}\right) \oplus J_{5}\left(\mu_{0}\right)$, 则

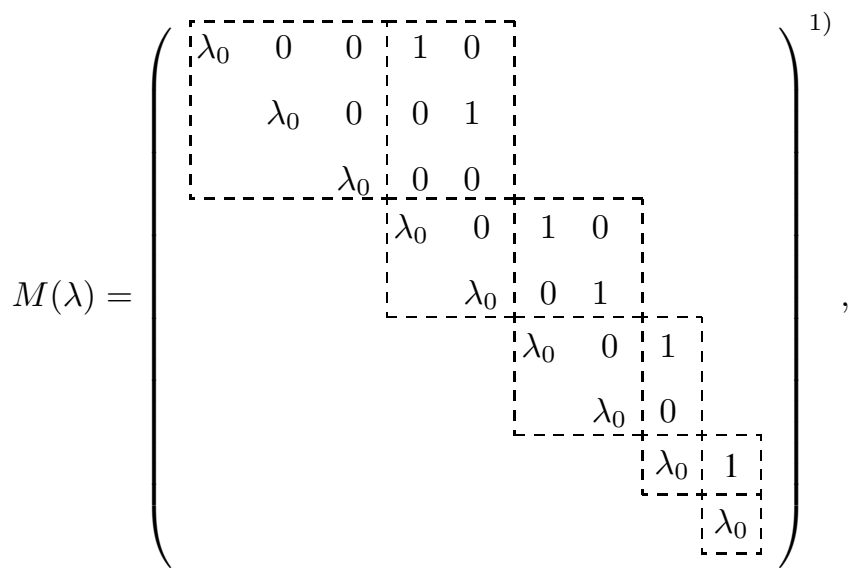

由关系 $S_{\mathcal{P}} M(\lambda)-M(\lambda) S_{\mathcal{P}}=0$ 可以得到

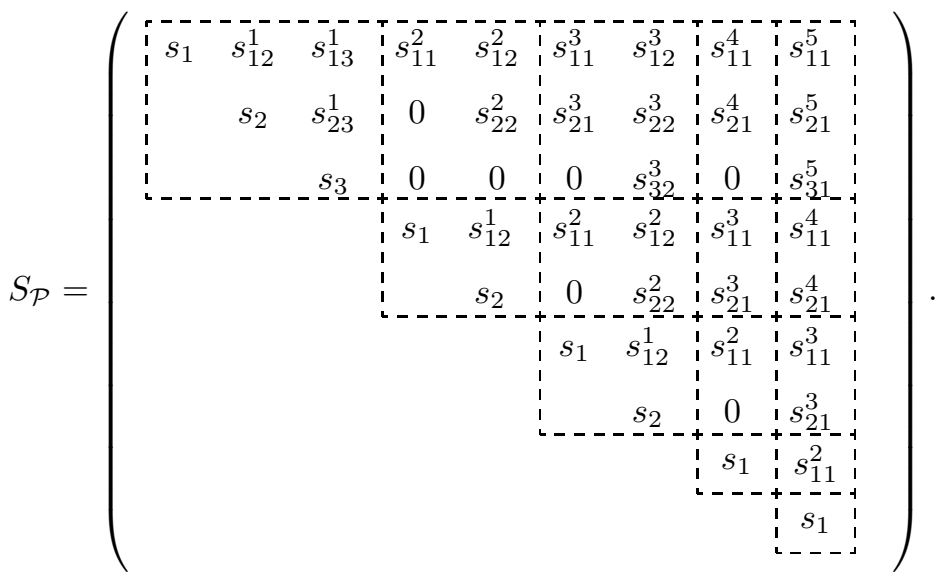

1) 这样的矩阵称为 Weyr 矩阵, 它能保证与它可交换的矩阵是上三角矩阵, 它可以由 Jordan 矩阵经 同时交换某些行、列而得到

www.scichina.com 
类似地,
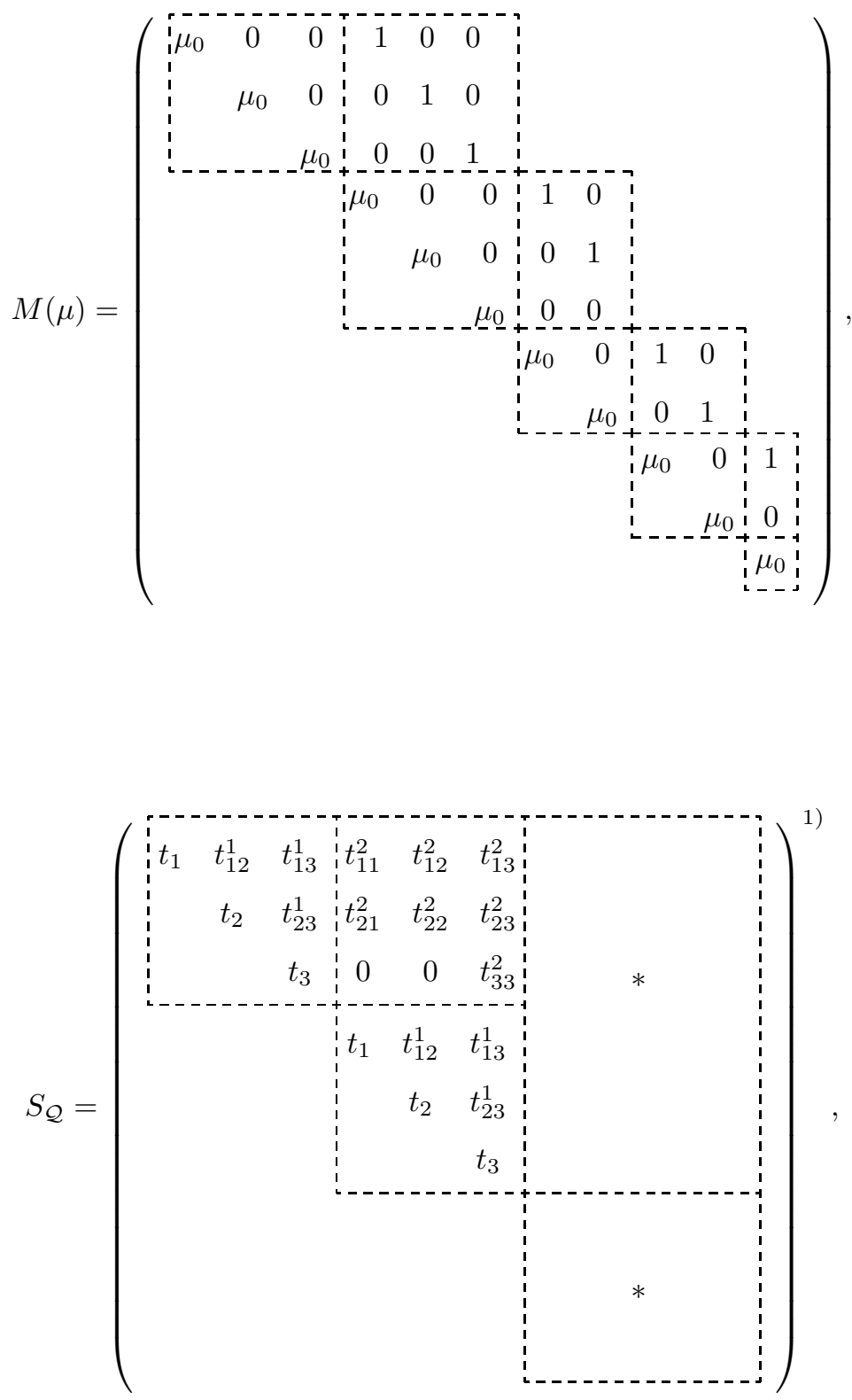

1) 为了方便, 我们仅写出矩阵中的某些重要元素, 0 由空格表示, 其他非零元素由 $*$ 表示 
则 $S\left(\delta\left(a_{1}\right)\right)$ 是一个如下形式的 $9 \times 11$ 矩阵:

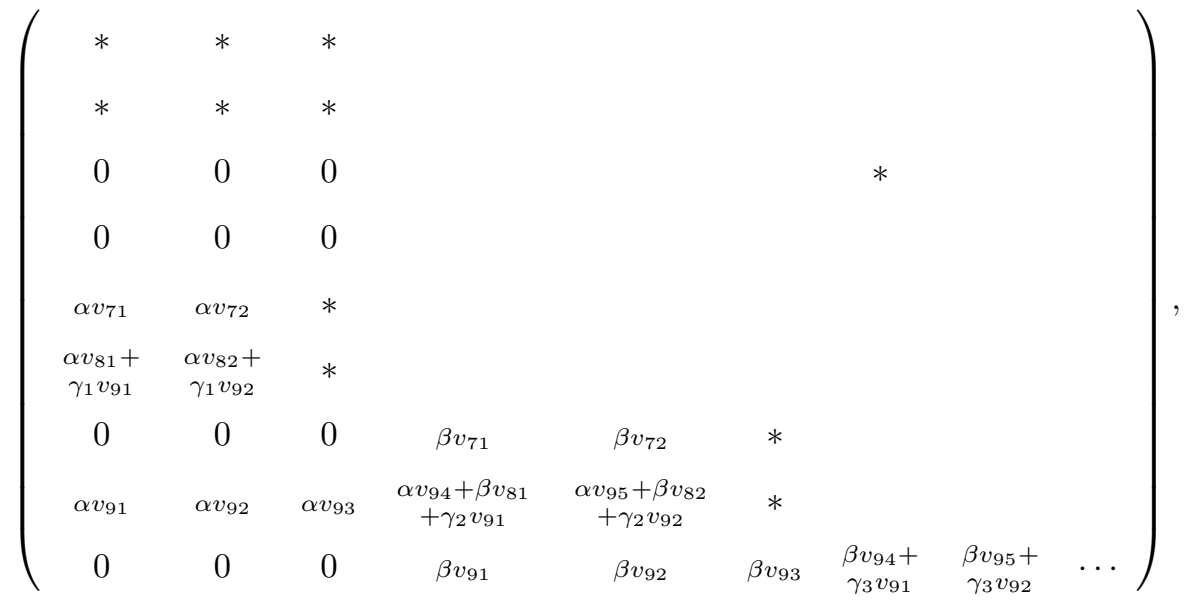

经 Belitskii 约化算法可得

$$
M\left(a_{1}\right)=\left(\begin{array}{lll}
0 & 0 & 0 \\
0 & 0 & 0 \\
1 & \xi & 0 \\
0 & 0 & 0 \\
\eta & 1 & 0 \\
1 & 0 & 0 \\
0 & 0 & 1 \\
0 & 0 & 1 \\
0 & 0 & 0
\end{array}\right.
$$

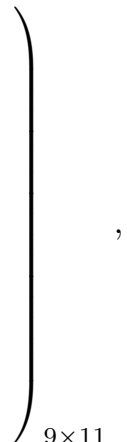

从而 $S_{\mathcal{P}}=s I_{9}+N_{1}, S_{\mathcal{Q}}=s I_{11}+N_{2}$, 其中 $N_{1}$ 和 $N_{2}$ 是上三角幕零矩阵, $s \in k$.

情形 $1(2), \alpha \neq 0$.

如果 $\gamma_{3} \neq 0, M(\lambda)$ 取与上一情形相同的矩阵, $M(\mu) \simeq J_{1}\left(\mu_{0}\right) \oplus J_{3}\left(\mu_{0}\right) \oplus$ $J_{5}\left(\mu_{0}\right)$, 则 $S_{\mathcal{Q}}$ 与 $S_{\mathcal{P}}$ 有相同的形式 (见上一情形);

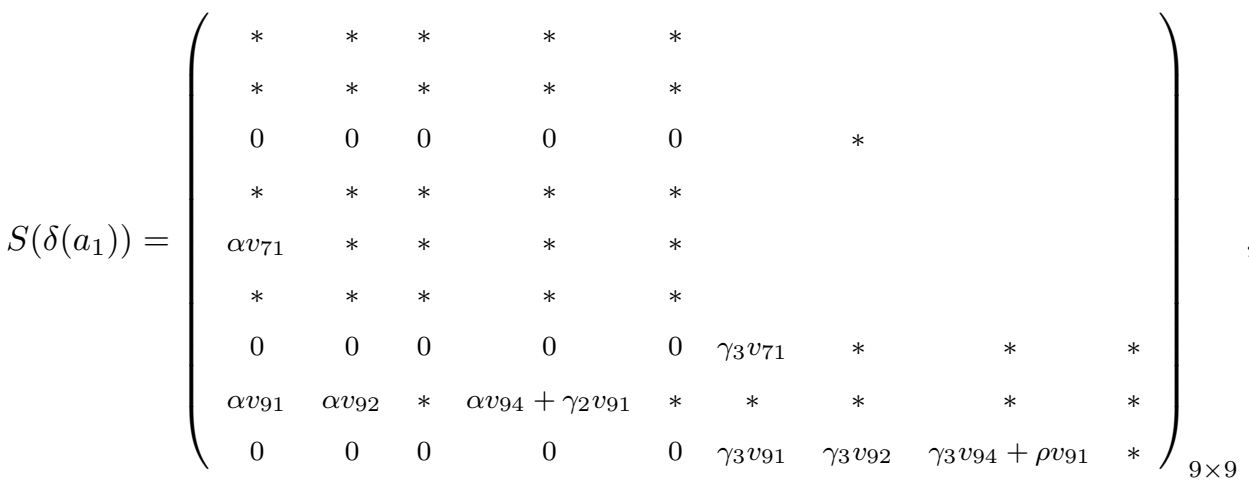

www.scichina.com 


$$
M\left(a_{1}\right)=\left(\begin{array}{llllll}
0 & 0 & 0 & 0 & 0 & \\
0 & 0 & 0 & 0 & 0 & \\
0 & 1 & 0 & \xi & 0 & \\
0 & 0 & 0 & 0 & 0 & 0 \\
\eta & 0 & 0 & 0 & 0 & 0 \\
0 & 0 & 0 & 0 & 0 & \\
0 & 0 & 1 & 0 & 1 & \\
0 & 1 & 0 & 1 & 0 & \\
0 & 0 & 0 & 0 & 0 &
\end{array}\right)_{9 \times 9} .
$$

情形 $1(2), \alpha \neq 0, \beta=0$ 且 $\gamma_{3}=0$; 或 $\alpha=\beta=0$. 令 $m_{\mathcal{P}}=9, m_{\mathcal{Q}}=4$,

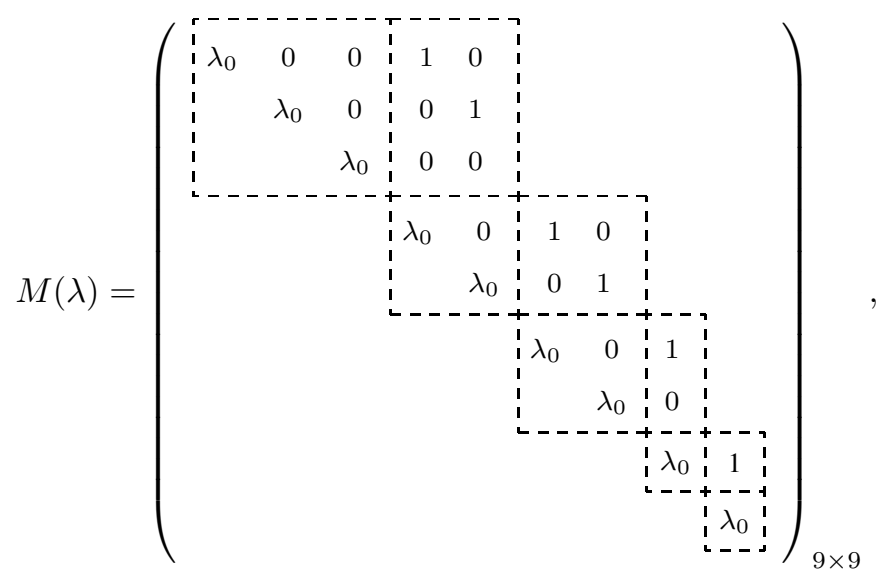

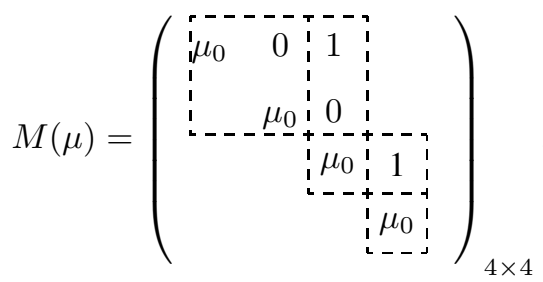

由关系 $S_{\mathcal{P}} M(\lambda)=M(\lambda) S_{\mathcal{P}}$ 可得

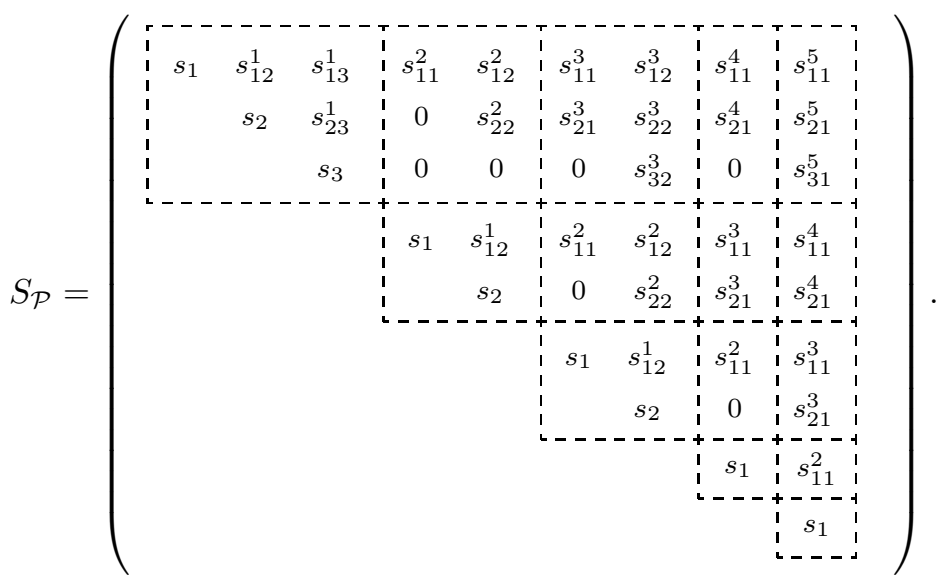

SCIENCE IN CHINA Ser. A Mathematics 
类似地, 由 $S_{\mathcal{Q}} M(\mu)=M(\mu) S_{\mathcal{Q}}$ 可得

$$
\begin{aligned}
& S_{\mathcal{Q}}=\left(\begin{array}{cccc}
y_{1} & y_{12}^{1} & y_{1}^{2} & y_{1}^{3} \\
& y_{2} & 0 & y_{21}^{3} \\
& & y_{1} & y_{1}^{2} \\
& & & y_{1}
\end{array}\right), \\
& \delta\left(a_{1}\right)=\left(\begin{array}{cccc}
* & * & * & * \\
* & * & * & * \\
0 & 0 & 0 & * \\
* & * & * & * \\
0 & 0 & 0 & * \\
* & * & * & * \\
0 & 0 & 0 & * \\
0 & 0 & 0 & * \\
0 & 0 & 0 & *
\end{array}\right), \quad \text { 取 } M\left(a_{1}\right)=\left(\begin{array}{cccc}
0 & 0 & 0 & 0 \\
0 & 0 & 0 & 0 \\
1 & \eta & \xi & 0 \\
0 & 0 & 0 & 0 \\
0 & 1 & 1 & 0 \\
0 & 0 & 0 & 0 \\
0 & 0 & 0 & 0 \\
1 & 0 & 0 & 0 \\
0 & 0 & 0 & 0
\end{array}\right) \text {, }
\end{aligned}
$$

则 $S_{\mathcal{P}}=s I_{9}+N_{1}, S_{\mathcal{Q}}=s I_{4}+N_{2}, s \in k, N_{1}, N_{2}$ 是上三角幕零矩阵.

情形 $1(1), \beta \neq 0$. 如果 $h(\lambda, \lambda)=0$, 或 $\operatorname{deg}(h(\lambda, \lambda))>0$, 则存在 $\lambda_{0} \in D$, 使得 $h\left(\lambda_{0}, \lambda_{0}\right)=0, f\left(\lambda_{0}\right) \neq 0$. 令 $m_{p}=4, M(\lambda) \simeq J_{1}\left(\lambda_{0}\right) \oplus J_{3}\left(\lambda_{0}\right)$,

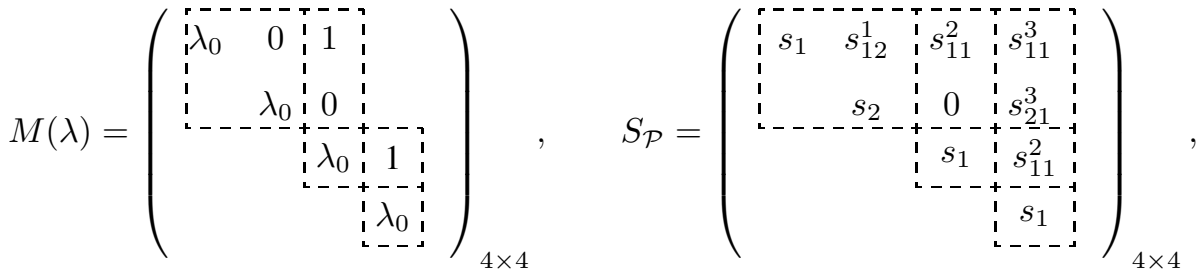

$$
\begin{aligned}
& S\left(\delta\left(a_{1}\right)\right)=\left(\begin{array}{cccc}
* & * & * & * \\
0 & * & * & * \\
\alpha v_{41} & \alpha v_{42} & * & * \\
0 & 0 & -\beta v_{41} & *
\end{array}\right)_{4 \times 4}, \quad M\left(a_{1}\right)=\left(\begin{array}{cccc}
0 & 0 & 0 & 0 \\
\xi & 0 & 0 & 0 \\
\eta & 0 & 0 & 0 \\
0 & 1 & 0 & 0
\end{array}\right)_{4 \times 4}
\end{aligned}
$$

从而 $S_{\mathcal{P}}=s I_{4}+N, N$ 是上三角幂零矩阵, $s \in k$.

情形 $1(1), \beta=0, \alpha \neq 0$.

$$
S\left(\delta\left(a_{1}\right)\right)=\left(\begin{array}{cccc}
* & * & * & * \\
0 & 0 & 0 & * \\
* & * & * & * \\
0 & 0 & 0 & *
\end{array}\right)_{4 \times 4} . \quad M\left(a_{1}\right)=\left(\begin{array}{cccc}
0 & 0 & 0 & 0 \\
\xi & 0 & 0 & 0 \\
0 & 0 & 0 & 0 \\
0 & 1 & \eta & 0
\end{array}\right),
$$

$M(\lambda) \simeq J_{1}\left(\lambda_{0}\right) \oplus J_{3}\left(\lambda_{0}\right)$ 取与上一情形相同的矩阵.

www.scichina.com 
情形 $1(1), \alpha=\beta=0$.

$$
S\left(\delta\left(a_{1}\right)\right)=\left(\begin{array}{cccc}
* & * & * & * \\
0 & 0 & 0 & * \\
0 & 0 & * & * \\
0 & 0 & 0 & *
\end{array}\right)_{4 \times 4} .
$$

$M(\lambda)$ 和 $M\left(a_{1}\right)$ 取与情形 $1(1), \beta=0, \alpha \neq 0$ 中相同的矩阵.

若对任意 $\lambda_{0} \in D, h\left(\lambda_{0}, \lambda_{0}\right) \neq 0$, 则必存在某个 $\lambda_{0} \neq \mu_{0}$ 满足 $h\left(\lambda_{0}, \mu_{0}\right)=0$, $f\left(\lambda_{0}\right) \neq 0, f\left(\mu_{0}\right) \neq 0$. 此时取 $\bar{M}(\lambda)=M(\lambda) \oplus M(\mu), \bar{M}\left(a_{1}\right)=\left(\begin{array}{cc}0 & M\left(a_{1}\right) \\ 0 & 0\end{array}\right)$, 其 中 $M(\lambda), M(\mu), M\left(a_{1}\right)$ 是情形 1(2) 中所构造的矩阵.

情形 2 . 令 $m_{\mathcal{P}}=5, m_{\mathcal{Q}}=2, M(\lambda)=J_{5}\left(\lambda_{0}\right), f\left(\lambda_{0}\right) \neq 0$, 则

$$
S_{\mathcal{P}}=\left(\begin{array}{ccccc}
s_{1} & s_{2} & s_{3} & s_{4} & s_{5} \\
& s_{1} & s_{2} & s_{3} & s_{4} \\
& & s_{1} & s_{2} & s_{3} \\
& & & s_{1} & s_{2} \\
& & & & s_{1}
\end{array}\right), \quad S_{\mathcal{Q}}=\left(\begin{array}{cc}
t_{11} & t_{12} \\
t_{21} & t_{22}
\end{array}\right), \quad M\left(a_{1}\right)=\left(\begin{array}{cc}
\xi & 0 \\
\eta & 0 \\
0 & 0 \\
1 & 0 \\
0 & 1
\end{array}\right),
$$

从而 $S_{\mathcal{P}}=s I_{5}, S_{\mathcal{Q}}=s I_{2}, s \in k$.

令矩阵 $M$ 中自由参数 $\xi$ 后面的元素全取 $0($ 矩阵元素的序取自下而上, 自左 而右), 则由上述构造知 $M \in R(\mathcal{A})$ 的自同态环是局部环, 因而是不可分解表示 (参见文献 $[7,8]$ ). 自由参数 $\eta$ 与 $\xi$ 是代数无关的, 其定义域是 $k^{2}$. 由注 3.3 知由 $M$ 诱导的函子 $F_{M}: k\langle x, y\rangle-\bmod \rightarrow R(\mathcal{A})$ 将

$$
L=\overbrace{}^{L(x)} k^{m} \bigcap^{L(y)}
$$

映为 $M^{m}(L(x), L(y))$, 该矩阵是将矩阵 $M$ 的常值元素 $a$ 扩大为 $a I_{m}$, 将自由参数 $\eta, \xi$ 分别改变为 $L(x)$ 和 $L(y)$ 而得到的. $M^{m}(L(x), L(y))$ 的自同态环由上三角分 块矩阵组成, 其对角块是 $\operatorname{End}_{k\langle x, y\rangle}(L)$, 因此诱导函子 $F_{M}$ 保持不可分解性与同 构类, 故 $\mathcal{A}$ 是野表示型的.

\section{4 可驯 bocs 的微分}

注意到定理 3.2 中所列出的极小野 bocs 是不完全的, 本节首先给出了一批 极小野 bocs, 并由此讨论了可驯 bocs 的微分.

引理 4.1 设 $\mathcal{A}=(A, V)$ 是一个 bocs, 其层为 $L=\left(A^{\prime} ; \omega ; a_{1} ; w_{1}, w_{2}\right), \operatorname{Obj} . A^{\prime}=$ $\{\mathcal{P}, \mathcal{Q}\}, A^{\prime}(\mathcal{P}, \mathcal{P})=k[\lambda], A^{\prime}(\mathcal{Q}, \mathcal{Q})=k[\mu], \delta\left(a_{1}\right)=h_{1}(\lambda) w_{1}+w_{2} h_{2}(\mu)$. 
则在下述情形下 $\mathcal{A}$ 必定是野表示型的:

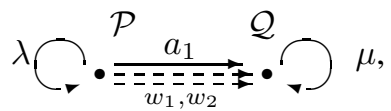

(1) $h_{1}(\lambda)=\left(\lambda-\lambda_{1}\right)\left(\lambda-\lambda_{2}\right), h_{2}(\mu)=\left(\mu-\mu_{1}\right)\left(\mu-\mu_{2}\right)$;

(2) $h_{1}(\lambda)=\left(\lambda-\lambda_{1}\right)\left(\lambda-\lambda_{2}\right)\left(\lambda-\lambda_{3}\right), h_{2}(\mu)=\mu-\mu_{1}$; 或对偶地, $h_{1}(\lambda)=\lambda-\lambda_{1}$, $h_{2}(\mu)=\left(\mu-\mu_{1}\right)\left(\mu-\mu_{2}\right)\left(\mu-\mu_{3}\right)$.

证 类似于定理 3.2 的证明, 只需构造 $\mathcal{A}$ 的不可分解自由参数化矩阵 $M$ 即 可.

(1) 如果 $\lambda_{1}=\lambda_{2}, \mu_{1}=\mu_{2}$, 令 $M(\lambda)$ 是与 $J_{1}\left(\lambda_{1}\right) \oplus J_{3}\left(\lambda_{1}\right)$ 相似的 Weyr 矩阵, $M(\mu)$ 是与 $J_{1}\left(\mu_{1}\right) \oplus J_{3}\left(\mu_{1}\right)$ 相似的 Weyr 矩阵; 如果 $\lambda_{1} \neq \lambda_{2}, \mu_{1}=\mu_{2}$ (或对偶的 情形), 令 $M(\lambda) \simeq J_{1}\left(\lambda_{1}\right) \oplus\left(J_{1}\left(\lambda_{2}\right) \oplus J_{2}\left(\lambda_{2}\right)\right), M(\mu) \simeq J_{1}\left(\mu_{1}\right) \oplus J_{3}\left(\mu_{1}\right)$ (特别地, 取其对偶); 如果 $\lambda_{1} \neq \lambda_{2}, \mu_{1} \neq \mu_{2}$, 令 $M(\lambda) \simeq J_{1}\left(\lambda_{1}\right) \oplus\left(J_{1}\left(\lambda_{2}\right) \oplus J_{2}\left(\lambda_{2}\right)\right), M(\mu) \simeq$ $J_{1}\left(\mu_{1}\right)^{2} \oplus\left(J_{1}\left(\mu_{2}\right) \oplus J_{2}\left(\mu_{2}\right)\right)$, 则 $M\left(a_{1}\right)$ 分别取

$$
\left(\begin{array}{llll}
0 & 0 & 0 & 0 \\
0 & 1 & \xi & 0 \\
0 & 1 & \eta & 0 \\
1 & 0 & 0 & 0
\end{array}\right), \quad\left(\begin{array}{llll}
1 & \eta & \xi & 0 \\
0 & 0 & 0 & 0 \\
0 & 1 & 1 & 0 \\
1 & 0 & 0 & 0
\end{array}\right), \quad\left(\begin{array}{lllll}
1 & \eta & 1 & \xi & 0 \\
0 & 0 & 0 & 0 & 0 \\
1 & 0 & 0 & 1 & 0 \\
0 & 1 & 1 & 0 & 0
\end{array}\right) .
$$

(2) 如果 $\lambda_{1}=\lambda_{2}=\lambda_{3}$, 令 $M(\lambda) \simeq J_{1}(\lambda) \oplus J_{4}(\lambda) \oplus J_{7}(\lambda), M(\mu) \simeq J_{1}\left(\mu_{1}\right)^{2} \oplus$ $J_{2}\left(\mu_{1}\right)$; 如果 $\lambda_{1}=\lambda_{2} \neq \lambda_{3}$, 令 $M(\lambda) \simeq\left(J_{1}\left(\lambda_{1}\right) \oplus J_{3}\left(\lambda_{1}\right)\right) \oplus\left(J_{1}\left(\lambda_{2}\right) \oplus J_{2}\left(\lambda_{2}\right)\right), M(\mu) \simeq$ $J_{1}\left(\mu_{0}\right)^{2} \oplus J_{2}\left(\mu_{0}\right)$; 若 $\lambda_{1}, \lambda_{2}, \lambda_{3}$ 两两不同, 则令 $M(\lambda) \simeq J_{1}\left(\lambda_{1}\right) \oplus\left(J_{1}\left(\lambda_{2}\right) \oplus J_{2}\left(\lambda_{2}\right)\right) \oplus$ $\left(J_{1}\left(\lambda_{3}\right) \oplus J_{2}\left(\lambda_{3}\right)\right), M(\mu) \simeq J_{1}\left(\mu_{0}\right)^{2} \oplus J_{2}\left(\mu_{0}\right)$, 那么 $M\left(a_{1}\right)$ 分别取

$$
\left(\begin{array}{llll}
0 & 0 & 0 & 0 \\
0 & 0 & 0 & 0 \\
0 & 1 & \xi & 0 \\
0 & 0 & 0 & 0 \\
0 & \eta & 0 & 0 \\
0 & 0 & 0 & 0 \\
0 & 1 & 0 & 0 \\
0 & 0 & 0 & 0 \\
1 & 0 & 1 & 0 \\
1 & 0 & 0 & 0 \\
0 & 0 & 0 & 0 \\
0 & 0 & 0 & 0
\end{array}\right),\left(\begin{array}{llll}
0 & 0 & 0 & 0 \\
0 & 1 & \xi & 0 \\
0 & \eta & 1 & 0 \\
1 & 1 & 0 & 0 \\
0 & 0 & 0 & 0 \\
0 & 0 & 1 & 0 \\
1 & 0 & 0 & 0
\end{array}\right),\left(\begin{array}{llll}
1 & \eta & \xi & 0 \\
0 & 0 & 0 & 0 \\
0 & 1 & 1 & 0 \\
1 & 1 & 0 & 0 \\
0 & 0 & 0 & 0 \\
0 & 0 & 1 & 0 \\
1 & 0 & 0 & 0
\end{array}\right) .
$$

www.scichina.com 
引理 4.2 设 $\mathcal{A}=(A, V)$ 是一个局部 bocs, 其层为 $L=\left(A^{\prime} ; \omega ; a_{1} ; w_{1}, w_{2}\right)$, Obj. $A^{\prime}=\{\mathcal{P}\}, A^{\prime}(\mathcal{P}, \mathcal{P})=k[\lambda], \delta\left(a_{1}\right)=h_{1}(\lambda) w_{1}+w_{2} h_{2}(\mu)$.

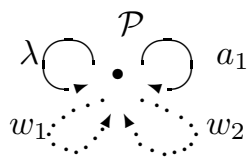

则在下述情形下 $\mathcal{A}$ 必定是野表示型的:

(1) $h_{1}(\lambda)=\left(\lambda-\lambda_{1}\right)\left(\lambda-\lambda_{2}\right), h_{2}(\mu)=\left(\mu-\mu_{1}\right)\left(\mu-\mu_{2}\right),\left\{\lambda_{1}, \lambda_{2}\right\} \cap\left\{\mu_{1}, \mu_{2}\right\}=\varnothing$;

(2) $h_{1}(\lambda)=\left(\lambda-\lambda_{1}\right)\left(\lambda-\lambda_{2}\right)\left(\lambda-\lambda_{3}\right), h_{2}(\mu)=\mu-\mu_{0},\left\{\lambda_{1}, \lambda_{2}, \lambda_{3}\right\} \cap\left\{\mu_{0}\right\}=\varnothing$, 或对偶的情形;

(3) $h_{1}(\lambda)=\left(\lambda-\lambda_{1}\right)\left(\lambda-\lambda_{2}\right), h_{2}(\mu)=\left(\mu-\lambda_{1}\right)$, 或对偶的情形.

证 对于情形 (1) 和 (2), 取 $\bar{M}(\lambda)=M(\lambda) \oplus M(\mu), \bar{M}\left(a_{1}\right)=\left(\begin{array}{cc}0 & M\left(a_{1}\right) \\ 0 & 0\end{array}\right)$ ， 其中 $M(\lambda), M(\mu), M\left(a_{1}\right)$ 是引理 4.1 中构造的矩阵.

(3) 若 $\lambda_{1}=\lambda_{2}$, 则令 $M(\lambda) \simeq J_{1}\left(\lambda_{1}\right) \oplus J_{3}\left(\lambda_{1}\right)$; 如果 $\lambda_{1} \neq \lambda_{2}$, 则令 $M(\lambda) \simeq$ $\left(J_{1}\left(\lambda_{1}\right) \oplus J_{2}\left(\lambda_{1}\right)\right) \oplus J_{1}\left(\lambda_{2}\right)$, 则 $M\left(a_{1}\right)$ 分别取

$$
\left(\begin{array}{llll}
0 & 0 & 0 & 0 \\
\xi & 0 & 0 & 0 \\
\eta & 0 & 0 & 0 \\
0 & 1 & 0 & 0
\end{array}\right), \quad\left(\begin{array}{llll}
0 & 0 & 0 & 0 \\
0 & \xi & 0 & 0 \\
\eta & 1 & 0 & 0 \\
1 & 0 & 0 & 0
\end{array}\right) .
$$

引理 4.3 设 $\mathcal{A}=(A, V)$ 是一个 bocs, 其层为 $L=\left(A^{\prime} ; \omega ; a_{1} ; w_{1}\right), \operatorname{Obj} . A^{\prime}=$ $\{\mathcal{P}, \mathcal{Q}\}, A^{\prime}(\mathcal{P}, \mathcal{P})=k[\lambda], A^{\prime}(\mathcal{Q}, \mathcal{Q})=k, \delta\left(a_{1}\right)=h(\lambda) w_{1}$ (或对偶的情形).

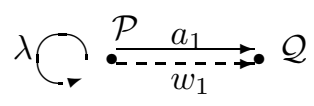

如果 $h(\lambda)=\left(\lambda-\lambda_{1}\right)^{e_{1}} \cdots\left(\lambda-\lambda_{r}\right)^{e_{r}}, \lambda_{1}, \cdots, \lambda_{r}$ 两两不同, $e_{i}$ 都是正整数且 $e_{1}+$ $\cdots+e_{r} \geqslant 4$, 那么 $\mathcal{A}$ 必定是野表示型的.

证 只需考虑 $e_{1}+\cdots+e_{r}=4$ 的情形. 该证明依赖于 4 的分划 $4=$ $\left(e_{1}, e_{2}, \cdots, e_{r}\right)$.

(4): $M(\lambda) \simeq J_{1}\left(\lambda_{1}\right) \oplus J_{4}\left(\lambda_{1}\right) \oplus J_{7}\left(\lambda_{1}\right)$;

$(3,1): M(\lambda) \simeq\left(J_{1}\left(\lambda_{1}\right) \oplus J_{4}\left(\lambda_{1}\right)\right) \oplus\left(J_{1}\left(\lambda_{2}\right) \oplus J_{2}\left(\lambda_{2}\right)\right) ;$

$(2,2): M(\lambda) \simeq\left(J_{1}\left(\lambda_{1}\right) \oplus J_{3}\left(\lambda_{1}\right)\right) \oplus\left(J_{1}\left(\lambda_{2}\right) \oplus J_{3}\left(\lambda_{2}\right)\right)$;

$(2,1,1): M(\lambda) \simeq\left(J_{1}\left(\lambda_{1}\right) \oplus J_{3}\left(\lambda_{1}\right)\right) \oplus\left(J_{1}\left(\lambda_{2}\right) \oplus J_{2}\left(\lambda_{2}\right)\right) \oplus J_{1}\left(\lambda_{3}\right) ;$

$(1,1,1,1): M(\lambda) \simeq\left(J_{1}\left(\lambda_{1}\right) \oplus J_{2}\left(\lambda_{1}\right)\right) \oplus\left(J_{1}\left(\lambda_{2}\right) \oplus J_{2}\left(\lambda_{2}\right)\right) \oplus J_{1}\left(\lambda_{3}\right) \oplus J_{1}\left(\lambda_{4}\right)$. 
$M\left(a_{1}\right)$ 分别取

$$
\left(\begin{array}{lll}
0 & 0 & 0 \\
0 & 0 & 0 \\
1 & \xi & 0 \\
0 & 0 & 0 \\
0 & 0 & 0 \\
0 & 0 & 0 \\
\eta & 0 & 0 \\
1 & 0 & 0 \\
0 & 0 & 1 \\
1 & 1 & 0 \\
0 & 0 & 1 \\
0 & 0 & 0
\end{array}\right), \quad\left(\begin{array}{lll}
0 & 0 & 0 \\
0 & 1 & \xi \\
0 & \eta & 1 \\
0 & 1 & 0 \\
1 & 0 & 0 \\
0 & 0 & 0 \\
0 & 1 & 0 \\
0 & 0 & 1
\end{array}\right),\left(\begin{array}{lll}
0 & 0 & 0 \\
0 & 1 & \xi \\
0 & \eta & 0 \\
1 & 1 & 0 \\
0 & 0 & 0 \\
1 & 0 & 0 \\
0 & 1 & 0 \\
0 & 0 & 1
\end{array}\right),
$$

由上述 3 个引理, 立即可得

定理 4.4 设 $\mathcal{A}=(A, V)$ 是一个层为 $L$ 的可驯 bocs, 它的第 1 个箭向是 $a_{1}$, 则 $\delta\left(a_{1}\right)$ 有如下可能:

(1)

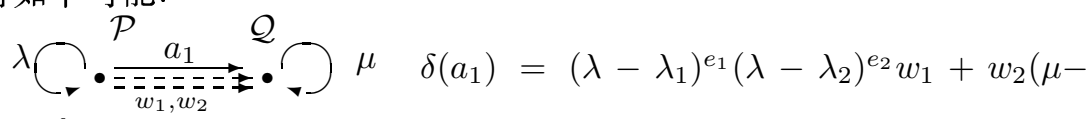
$\left.\mu_{1}\right)^{f_{1}}\left(\mu-\mu_{2}\right)^{f_{2}}, e_{1} f_{1} \neq 0, e_{1}+e_{2}+f_{1}+f_{2} \leqslant 3, w_{1}$ 与 $w_{2}$ 是线性无关的, $\left(\lambda_{1}, \mu_{1}\right)$, $\left(\lambda_{2}, \mu_{2}\right) \in D$ 由引理 4.1 给出.

(2)

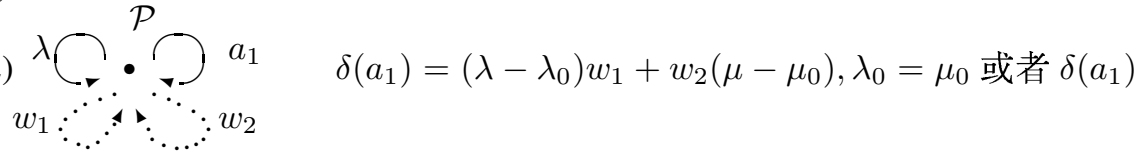
如情形 (1), $\left\{\lambda_{1}, \lambda_{2}\right\} \cap\left\{\mu_{1}, \mu_{2}\right\}=\varnothing, w_{1}$ 与 $w_{2}$ 是线性无关的.

(3) $\lambda \bigcirc \stackrel{\mathcal{P} a_{1}}{--\bar{w}_{1}^{--} \bullet} \mathcal{Q} \delta\left(a_{1}\right)=\left(\lambda-\lambda_{1}\right)^{e_{1}}\left(\lambda-\lambda_{2}\right)^{e_{2}}\left(\lambda-\lambda_{3}\right)^{e_{3}} w_{1}, \lambda_{1}, \lambda_{2}, \lambda_{3}$ 两两不同, $1 \leqslant e_{1}+e_{2}+e_{3} \leqslant 3$, 或对偶的情形.

(4) $a_{1} \underset{\mathrm{C}}{\mathrm{P}}$ 或 $\underset{\mathcal{P}}{\stackrel{Q}{\longrightarrow}} a_{1}$

(5) 对以上情形, $\delta\left(a_{1}\right)=w_{1}$.

致谢 感谢审稿人提出的宝贵意见 


\section{参考文献}

1 Drozd Yu A. Tame and wild matrix problems. In: Representations and Quadratic forms. Inst Math Akad Nauk Ukrain SSR, Kiev, 1979: 39 74 (in Russian)

2 Roiter A V. Matrix problems and representations of BOCS's. In: Dlab V, Gabriel P, eds. Representation Theory I, LNM 831. Berlin: Springer-Verlag, 1980. 288 324

3 Crawley-Boevey W W. On tame algebras and bocses. Proc London Math Soc, 1988, 56(3): 451 483

4 Zeng Xiangyong, Zhang Yingbo. A correspondence of almost split sequences between some categories. Comm Alg, 2001, 29(2): 1 26

5 Crawley-Boevey W W. Matrix problems and Drozd's theorem. In: Balcerzyk S, et al eds. Topics in Algebra. Banach Center Publications, Vol 26, Part 1. Warsaw: PWN-Polish Scientific Publishers, 1990. 199 222

6 Bautista R, Kleiner M. Almost split sequences for relatively projective modules. J Alg, 1990, 135(1): $19 \sim 56$

7 Sergeichuk V V. Canonical matrices for basic matrix problems. Linear Algebra and its Applications, 2000, 317(1/3): 53 102

8 徐运阁, 张英伯. 不可分解性与链环数. 中国科学, A 辑, 2001, 31(5): 385 391 